\title{
Development of Single Phase Heat Transfer Correlations for Water \& R134a in Rectangular Channel with Smooth Wavy Fin
}

\author{
Amaranatha Raju. Muppala ${ }^{1 *}$, Ashok Babu T. $\mathrm{P}^{2}$ and Ranganayakulu.Chennu ${ }^{1}$ \\ 1. Aeronautical Development Agency, PB No.1718, Vimanapura post, Bangalore 560017, India \\ 2. Department of Mechanical Engg, NITK, Surathkal, Mangalore 575025, India
}

\begin{abstract}
Single phase heat transfer analysis of water and R134a refrigerant (liquid phase) has been carried out using CFD (Computational fluid dynamics) approach for rectangular channel with smooth wavy fin. Colburn $j$ factor and Fanning friction factor $f$ are predicted for wavy fin. The correlations are developed at Reynolds number range of 100-15,000. The effect of fin geometry (fin spacing, fin height, wave height and wave length) on the enhanced heat transfer and pressure drops are investigated. Results show that there is no significant variation of $f$ factor for water and liquid R134a at constant Reynolds number. However variations in $j$ factor were observed at constant Reynolds number. Colburn $j$ factor and Fanning friction factor $f$ correlations are proposed in terms of Re and geometry parameters $(\mathrm{h} / \mathrm{s}, \mathrm{a} / \mathrm{s}, \lambda / \mathrm{a})$ for water and liquid refrigerant $\mathrm{R} 134 \mathrm{a}$ in the present study. Two separate equations are proposed for the low and high Re regions i.e. between Re of 100-1,000 and Re of 1,000-15,000.
\end{abstract}

Key words: Compact heat exchanger, numerical analysis, friction factor, Colburn factor, wavy fin, lance \& offset fin, heat transfer coefficient.

\section{Nomenclature}

$\begin{array}{ll}\mathrm{A} & \text { Total heat transfer area, } \mathrm{m}^{2} \\ \mathrm{~A}_{\mathrm{s}} & \text { Fins surface area in channel, } \mathrm{m}^{2} \\ \mathrm{a} & \text { Wave height, } \mathrm{mm} \\ \mathrm{D}_{\mathrm{h}} & \text { Hydraulic diameter, } \mathrm{m} \\ f & \text { Fanning friction factor, dimensionless } \\ \mathrm{G} & \text { Mass flux, } \mathrm{kg} / \mathrm{m}^{2} \mathrm{~s} \\ \mathrm{f}_{\mathrm{h}} & \text { Fin height, } \mathrm{m} \\ \mathrm{h} & \text { Fin height, } \mathrm{mm} \\ j & \text { Colburn factor, dimensionless } \\ \mathrm{k} & \text { Thermal conductivity of fin, } \mathrm{W} / \mathrm{m} \mathrm{K} \\ \dot{m} & \text { Mass flow rate, } \mathrm{kg} / \mathrm{s} \\ \mathrm{Nu} & \text { Nusselt number, dimensionless } \\ \mathrm{Pr} & \text { Prandtl number, dimensionless } \\ \Delta \mathrm{P} & \text { Pressure drop, Pa } \\ \mathrm{q} & \text { Heat flux, } \mathrm{W} / \mathrm{m}^{2} \\ \mathrm{Re} & \text { Reynolds number, dimensionless } \\ \mathrm{s} & \text { Lateral fin spacing, mm } \\ \mathrm{T} & \text { Temperature, }{ }^{\circ} \mathrm{C}\end{array}$

Corresponding Author: Amaranatha Raju. Muppala M. E, scientist, research fields: heat transfer, heat exchangers and boiling. $\quad$ E-mail: amaranatha@yahoo.com; raju@jetmail.ada.gov.in.

$\begin{array}{ll}\mathrm{t} & \text { Fin thickness, } \mathrm{mm} \\ \lambda & \text { Wave length, } \mathrm{mm}\end{array}$

\begin{tabular}{|c|c|}
\hline \multicolumn{2}{|c|}{ Greek Symbols } \\
\hline$\Phi$ & Generalized transport variable \\
\hline$\rho$ & Density, $\mathrm{kg} / \mathrm{m}^{3}$ \\
\hline$\kappa$ & Turbulent kinetic energy, $\mathrm{m}^{2} / \mathrm{s}^{2}$ \\
\hline$\Gamma$ & Effective diffusivity, $\mathrm{m}^{2} / \mathrm{s}^{2}$ \\
\hline$\varepsilon$ & Turbulent dissipation rate, $\mathrm{m}^{2} / \mathrm{s}^{2}$ \\
\hline$\mu$ & Dynamic viscosity, Ns $/ \mathrm{m}^{2}$ \\
\hline
\end{tabular}

\section{Introduction}

Compact plate fin heat exchangers have become popular in cooling and heating applications across industries especially in auto mobile, aerospace and electronics. The demand for miniaturization of components is high in these industries to reduce weight, size and save the cost. Due to its high effectiveness compact heat exchangers will have low weight and occupy less space compared to other type of heat exchangers. London and Shah [1] has defined 
"Compact heat exchanges as one having an area density greater than $700 \mathrm{~m}^{2} / \mathrm{m}^{3}$ ". Such compactness is achieved by providing a high density fins in the flow passage. There are different types of fins like offset fins, wavy fins, plain fins, perforated fins, pin fins and louvered fins, which are to provide the high density area and to enhance the heat transfer coefficient in compact heat exchangers. The most common among them are lance \&_offset and wavy fins. Predicted or measured dimensionless parameters (Colburn $j$ factor and Fanning friction $f$ vs. Reynolds number Re) of these fins are essential for design of heat exchangers. Kays and London [2] conducted the experiments and compiled the results for 3 wavy fins and 21 lance $\&$ offset fins. Joshi and Webb [3] developed empirical correlations for heat transfer coefficient and friction factor in the offset strip-fin heat exchanger surface geometry. Friction factor data were taken on eight scaled-up, idealized geometries. Wieting [4] developed empirical relations by correlating experimental heat transfer and flow friction data for 22 offset strip fin surfaces over two Reynolds number ranges $\mathrm{Re}_{\mathrm{d}}<$ 1,000 and $\operatorname{Re}_{\mathrm{d}}>2,000$. For predicting $j$ and $f$ factors in the transition region $\left(1,000<\operatorname{Re}_{\mathrm{d}}<2,000\right)$, he suggested extrapolating the equations up to their intersection point. Manglik and Bergles [5] used an asymptotic method to establish their correlations for offset strip fin surfaces. They have considered 18 offset fin surfaces and analyzed for heat transfer and friction data. They also have analyzed effect of fin geometry parameters and proposed correlations in terms of geometry parameters. Ranganayakulu and Pallavi [6] carried out numerical analysis on offset fins using air as a media and proposed correlations. They have analyzed the fin geometry on $j$ and $f$ factors. Zhang et al. [7]. numerically simulated the two dimensional wavy plate fin channels with sinusoidal wall corrugations at low Reynolds number for air and presented velocity and temperature fields, isothermal Fanning friction factor $f$, and Colburn $j$ factor for different flow rates $(10 \leq \mathrm{Re} \leq$ $1,000)$, wall-corrugation severity $(0: 125 \leq \gamma \leq 0: 5)$, and fin spacing $(0: 1 \leq \varepsilon \leq 3: 0)$. Awad and Muzychka [8] proposed the new models for prediction of Fanning friction factor $f$ and Colburn $j$ factor for air cooled compact wavy fin heat exchangers. The new models are developed by combining the asymptotic behavior for the low Reynolds number and laminar boundary layer regions. The models are developed by taking into account the geometric variables such as fin height, fin spacing, wave amplitude, fin wavelength, Reynolds number, and Prandtl number. They have compared the models with numerical and experimental data for air at different values of the geometric variables.

All the studies carried out above used air as the coolant medium. Only few studies performed using liquid as coolant. Even though for liquid cooling these fins has been used for many years in applications, adequate data is not available in the open literature. Since the liquids have better heat transfer coefficient than the air, liquids are preferred to air in some applications. Hu and Herold [9, 10] carried out studies both analytically and experimentally using liquid water and polyalphaolefin for Prandtl number ranges from 3 to 150 on offset fin surface. They have presented that the Prandtl number had a significant effect on the heat transfer factor $j$ of the fin. They also observed that air models over predict the $j$ factor for liquids. However the Prandtl number was found to have little on the Fanning friction factor. Ranganayakulu et al. [11] performed numerical analysis on offset fins using CFD (Computational fluid dynamics) approach. He found that the $j$ of water is lower by about $30 \%$ when compared with air, where as there is no significant deviation in $f$ values. Even though $j$ is lower for water, the heat transfer coefficient is much higher when compared to air. Kim and Sohn [12] carried out experiments using water and R113 as a test fluid on Offset strip fins by considering fin geometry effect. In their experimental findings noted that there is no significant variation of $f$ factor. However, the Colburn factor measured in their study was about $25 \%$ smaller than the prediction of Manglik and Bergles for $\mathrm{Re}<$ 
1,000. For $\operatorname{Re}>1,000$, the difference between the experimental data and the prediction of Manglik and Berglesreduced as the Reynolds number increased.

Even though few studies performed analysis on Offset fins using liquid medium, there is no data available on wavy fin surfaces using liquid medium, in the open literature. The experimental results of Kays and London on three wavy fins are only the data available in open literature. But they have used air as a medium. Maiti [13] carried out numerical analysis on wavy fin using air as medium and proposed correlations. Ranganayakulu et al. [14] carried out numerical study of flow patterns of compact plate fin heat exchangers and generation of design data for wavy fins using CFD for air as a coolant and proposed correlations for $f$ and $j$ by considering the geometry of fins.

Since ample data is not available in open literature on liquid cooled wavy fin heat exchangers, the present work has been initiated. The main objective of this study is to find out single phase heat transfer coefficient of water and R134a on wavy fin and effect of geometry parameters using numerical analysis, since single phase heat transfer data is not available in open literature for wavy fins. This data shall be useful reduce the experimental study on heat transfer and friction factors for conducting experiments on two phase heat transfer analysis since two phase heat transfer coefficient is related with single phase heat transfer coefficient as proposed by Chen [15]. Chen proposed that the boiling heat transfer coefficient on the refrigerant $\left(\mathrm{h}_{\mathrm{r}}\right)$, is sum of the convective term $\left(\mathrm{h}_{\mathrm{cb}}\right)$, and the nucleate boiling term $\left(\mathrm{h}_{\mathrm{nb}}\right)$.

$$
h_{r}=h_{c b}+h_{n b}
$$

The convective heat transfer coefficient $\left(h_{c b}\right)$ is related with single-phase heat transfer coefficient $\left(\mathrm{h}_{\mathrm{f}}\right)$ by the Reynolds number factor $(F)$.

$$
h_{c b}=F h_{f}
$$

\section{Methodology}

The following steps are carried out in numerical analysis for generation of Colburn $j$ factor and friction factor $f$ for wavy fin using liquid water and liquid R134a: Modeling and numerical grid generation for the fin; Applying the boundary conditions; Analyzing the fins using ANSYS (Analysis system) CFD software; Computation of $j$ and $f$ factors and validation of results with standard values and Establishing correlations.

\subsection{Mathematical Model}

Following are some of the assumptions made in the CFD simulation:

(a) The flow is study and incompressible.

(b) The fluid flow meets the Boussinesq assumption.

(c) The effect of heat conduction through the fin and plate material are considered.

(d) Air, liquid water and liquid R134a are used as working medium.

In this work, the CFD software ANSYS CFD is employed for simulation. In ANSYS CFD, the conservation equations of mass, momentum and energy are solved using the finite volume method. There are several turbulence models available in the code. The turbulent flow is calculated by the semi-implicit SIMPLER as mentioned in Versteeg and Malalasekera [16] Algorithm method in the velocity and pressure conjugated problem, and a second order upwind differential scheme is applied for the approximation of the convection terms.

A standard $\kappa-\varepsilon$ model as given in Versteeg and Malalasekera [16] with enhanced wall treatment is used to predict turbulent flow in the plate-fin heat exchanger as well as in the fin geometry. The Reynolds transport equations can be written in a generalized form as given in [17, 18].

$$
\operatorname{div}(\rho u \Phi)=\operatorname{div}(\Gamma \operatorname{grad} \Phi)+\mathrm{S}_{\Phi}
$$

where, $\Phi$ stands for a generalized transport variable, which is used for all conserved variables in a fluid flow problem, including mass, momentum and the turbulence variables $\kappa$ and $\varepsilon$. $\Gamma$ represents the effective diffusivity (sum of the eddy diffusivity and the molecular diffusivity). $\mathrm{S}_{\Phi}$ is the source term for the 
respective dependent variable. The solution of the above set of equations is applied to the prediction of velocity and turbulence levels throughout the domain. The convergent criteria is specified to absolute residuals $\left(\leq 1.0 \times 10^{-5}\right)$.

\section{Computational Domain, Boundary Conditions and Numerical Model}

Wavy fin is one of the most popular fin surfacesin plate fin heat exchangers; it can lengthen the flow path, creates turbulence and promotes better mixing. They are uninterrupted surfaces with cross-sectional shape similar to that of plain fins except for the undulations in the flow direction. The heat transfer and flow friction performance of wavy fin surfaces lies between those of plain fin and offset strip fins.

Numerical model has been developed by considering geometrical parameters. The geometry of the smooth wavy fin is defined by the following parameters.

Fin thickness $(t)$, fin height $(h)$, wave length $(\lambda)$, wave amplitude (a), fin spacing (s).

Fig. 1 shows the schematic view of the smooth wavy fin surface and defines the geometric parameters.

The following set of dimensionless parameters is defined for the wavy fin, largely expressing heat transfer and flow friction data for deriving correlations. The effect of fin thickness is neglected in the analysis. The geometry parameters are expressed in terms of $\alpha, \beta$ and $\gamma$ for convenience.

$$
\begin{aligned}
& \alpha=\mathrm{h} / \mathrm{s} \\
& \beta=\mathrm{a} / \mathrm{s} \\
& \gamma=\lambda / \mathrm{a}
\end{aligned}
$$

Hydraulic diameter $\mathrm{D}_{\mathrm{h}}$ is used as the characteristic dimension in expressing the heat transfer and flow friction data. The hydraulic diameter $\mathrm{D}_{\mathrm{h}}$ is used to define the Reynolds number, computational domain, boundary conditions and numerical model.

Fig. 2 shows the computational domain taken for modeling fluid flow and heat transfer over a smooth wavy surface. Fin spacing $\mathrm{s}$ in the $\mathrm{x}$-direction, wave length $\lambda$ in the $y$-direction and fin height in the z-direction constitutes the computational domain.

A total of 28 sets wavy fin surfaces are considered and modeled for CFD analysis for developing the correlations. Two fluids liquid water and refrigerant $\mathrm{R}$ 123a (liquid phase) are used as medium for the analysis. The following ranges of fin geometries and Reynolds numbers are taken for analysis.

$$
\begin{gathered}
2.83 \leq \mathrm{h} / \mathrm{s} \leq 11.24 \\
1.13 \leq \mathrm{a} / \mathrm{s} \leq 2.53 \\
4.14 \leq \lambda / \mathrm{a} \leq 8
\end{gathered}
$$

$100 \leq \operatorname{Re} \leq 1,000$ (laminar region)

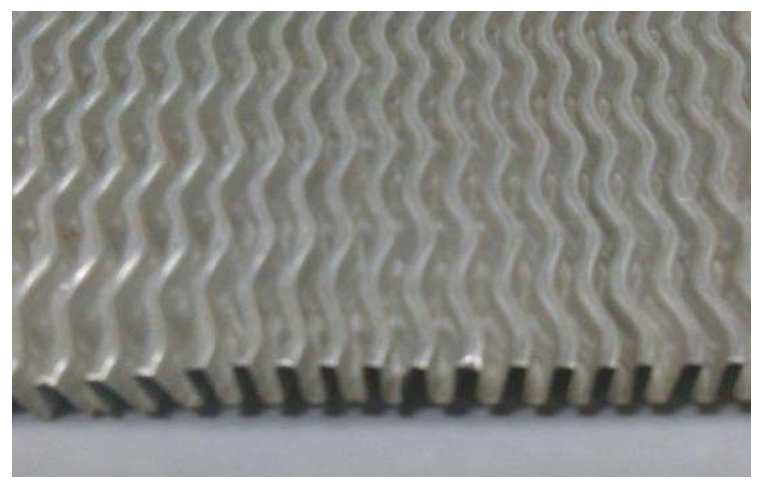

(a)

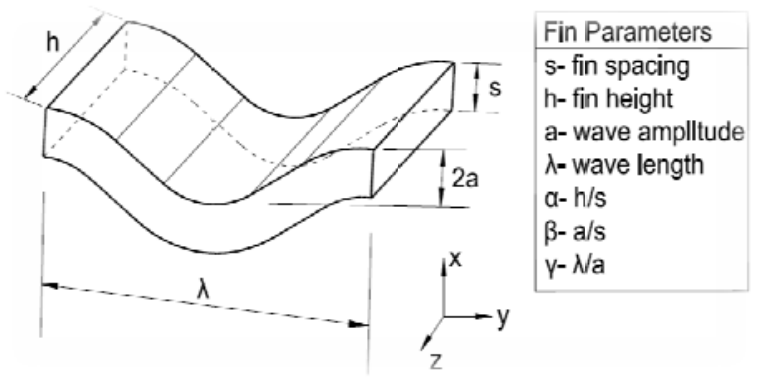

(b)

Fig. 1 (a) Wavy fin surface and (b) geometry of a typical smooth wavy fin surface.

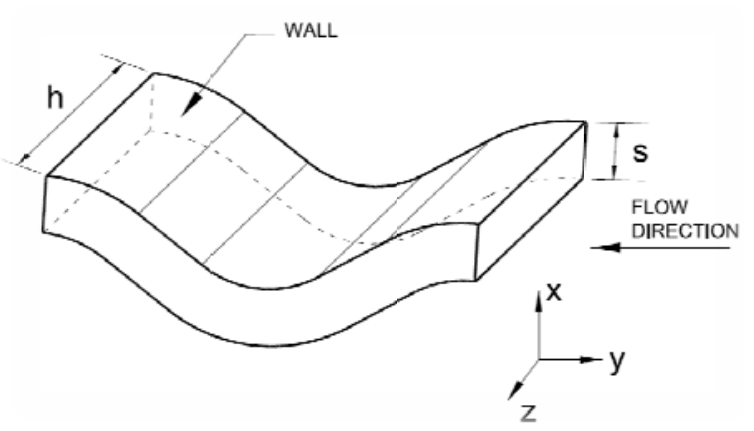

Fig. 2 The computational fluid domain for the smooth wavy fin surface. 


$$
1,000 \leq \operatorname{Re} \leq 15,000 \text { (turbulent region) }
$$

The following Boundary conditions are used while analyzing the models:

(a) No slip condition.

(b) Constant wall temperature.

(c) Periodicity condition.

(d) Total flow at the inlet.

(e) Bulk temperature of fluid at inlet.

(f) Constant pressure boundary condition.

\section{CFD Approach}

The CFD approach is carried out using ANSYS CFD for an estimation of $j$ and $f$ factors for different smooth wavy fin geometry using water and liquid refrigerant R134a as a medium. In this model, a single layer of actual wavy fin is modeled and generated grid. The three-dimensional computational domain of this model is shown in Fig. 2. CFD analysis is carried out for different fin geometries and Reynolds number as mentioned above. Before carrying out the actual analysis grid independence check was done to determine the grid size. Fig. 3 shows grid independence curve drawn between the number of elements and pressure drop parameters. The mesh size is determined based upon the curve where the slope is almost zero.

The CFD analysis was carried out in two phases. In first phase the fin characterized for $f$ values over range of Reynolds number. In second phase the $j$ value is estimated for the same range of Reynolds number using energy equation. In order to overcome the entrance effect, the concept of periodic fully developed flow as suggested by Patankar et al. [19] is implemented for the flow analysis. The pressure drop for unit length is estimated from ANSYS CFD initially and is used for estimation of the friction factor $f$ as per Kays and London [2] procedure. Similarly, the procedure is repeated for the range of Reynolds numbers from 100 to 15,000 in order to draw the effect of Reynolds number on friction factor.

In the second phase the "velocity inlet" and "outflow (pressure outlet)" boundary conditions are used at the inlet and outlet of the fin geometry, respectively. This analysis takes heat conduction into account. Weimer and Hartzog [20] and Hasseler [21] suggested a simplified assumption of the constant wall temperature boundary condition over all section of the fin. The temperature difference between inlet and outlet of the fin, in turn, is used for calculating the $j$ factor using the Kays and London [2] methodology. Similarly, the same procedure is repeated for the range of Reynolds numbers from 100 to 15,000 in order to draw the $j$ versus Re characteristic curve.

\section{Validation}

To validate the CFD results, Offset fin geometry from Kim and Sohn [12] has been taken for the CFD analysis and modeled, since experimental results with wavy fin and liquid water/refrigerant R134a as working medium is not available in the open literature. The offset fin geometry considered for the analysis is

Fin height, $\mathrm{h}(\mathrm{mm}) 2.8$, fin length, $l(\mathrm{~mm}) 1.5$, lateral fin spacing, $\mathrm{s}(\mathrm{mm}) 3.5$, fin thickness, $\mathrm{t}(\mathrm{mm}) 0.2$ and hydraulic diameter, $\mathrm{D}_{\mathrm{h}}(\mathrm{mm}) 2.84$.

The above fin geometry was modeled; grid generated and carried out CFD analysis using ANSYS CFD. The results were analyzed and compared with experimental results of Kim and Sohn [12] and plotted

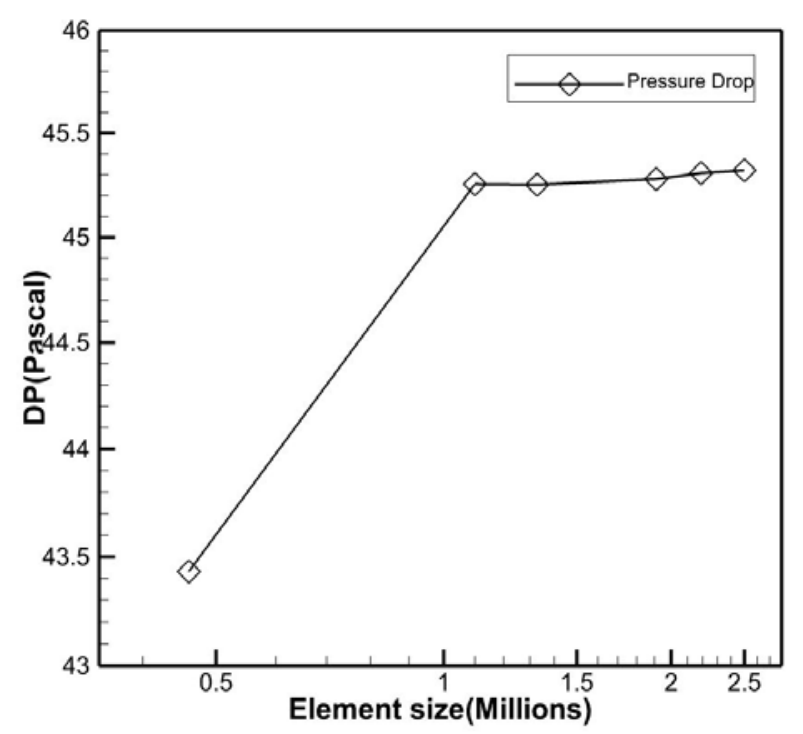

Fig. 3 Grid independency graph. 
in Fig. 4. The CFD results are found in good agreement with experimental results. The variation is found to less than $5 \%$ in both $f$ and $j$ values. Uniform wall temperature boundary conditions are used for the analysis. Before carrying out the analysis grid independence check was carried out to optimize the mesh size.

The CFD results also were validated with wavy fin and air medium, by considering the wavy fin geometry from Kays \& London [2]. The wavy fin geometry was considered for the analysis: Fin height, h (mm) 6.77,

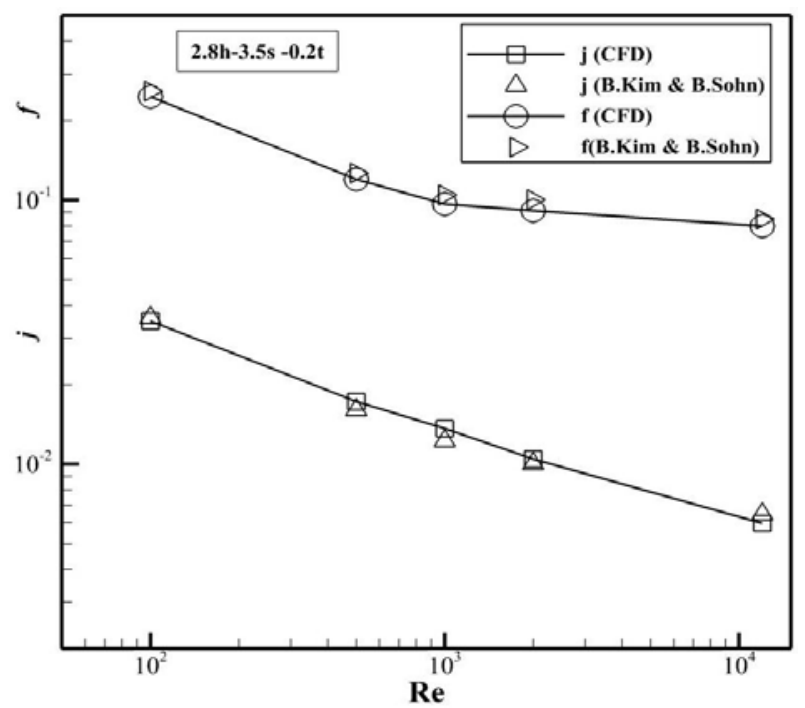

Fig. 4 Validation with experimental results for the fin 2.8 h-3.5 s-0.2 t.

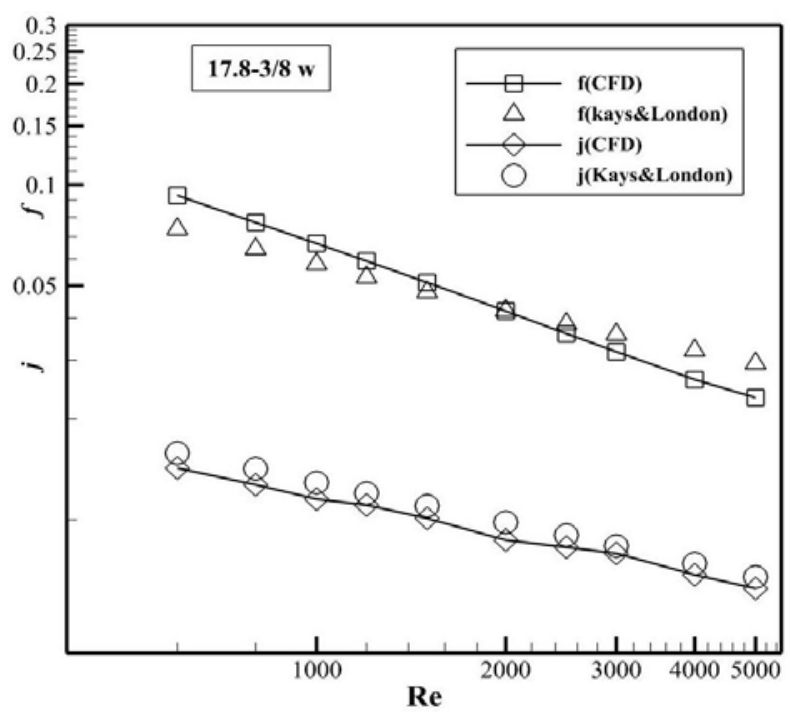

Fig. 5 Validation with experimental results for the fin 6.77 h-1.411 s-0.152 t. wave length, $l(\mathrm{~mm}) 9.525$, fin spacing, $\mathrm{s}(\mathrm{mm}) 1.411$, fin thickness, $\mathrm{t}(\mathrm{mm})$ 0.152, wave amplitude, $\mathrm{a}(\mathrm{mm})$ 1.95 and hydraulic diameter, $\mathrm{D}_{\mathrm{h}}(\mathrm{mm}) 2.123$.

The above fin geometry was modeled; grid generated and carried out CFD analysis using ANSYS CFD. The results were analyzed and compared with experimental results of Kays \& London [2] and plotted in Fig. 5. The CFD results were found in good agreement with experimental results. The variation was found to less than $5 \%$ in both $f$ and $j$ values. Uniform wall temperature boundary conditions were used for the analysis. Before carrying out the analysis grid independence check was carried out to optimize the mesh size.

\section{Results and Discussion}

CFD analysis for smooth wavy fin surface is carried out using ANSYS CFD at various Reynolds numbers with the boundary conditions. From the Figs. 4 and 5, it is observed that $f$ and $j$ vs. Re curves of wavy fin follow the same trend as Kim \& Sohn [12] and Kays \& London [2] experimental results. Hence the CFD analysis has been extended to smooth wavy fin under study. The effects of variations of dimensionless parameters on wavy fin surface performance are presented. The response of velocity, pressure and temperature fields to changes in geometric parameters and Reynolds number is clearly manifested. Figs. 6-8 show the role of geometric parameters $\mathrm{h} / \mathrm{s}, \mathrm{a} / \mathrm{s}, \lambda / \mathrm{a}$ vs. $f$ and parameters $\mathrm{h} / \mathrm{s}, \mathrm{a} / \mathrm{s}, \lambda / \mathrm{a}$ vs. $j$ in determining the heat transfer and flow friction performance of smooth wavy fin surfaces. The individual effects geometrical parameters variations are explained in the following paragraphs.

\subsection{Effect of h/s Ratio on $j$ and $f$}

The friction factor $f$ and Colburn $j$ factors are plotted against the fin height (h)-to- fin spacing (s) ratio (h/s) for varying Reynolds number in Fig. 6 for water and liquid refrigerant R134a. Both $f$ and $j$ decrease with increase in $\operatorname{Re}$ as expected. Friction factor $f$ increase 


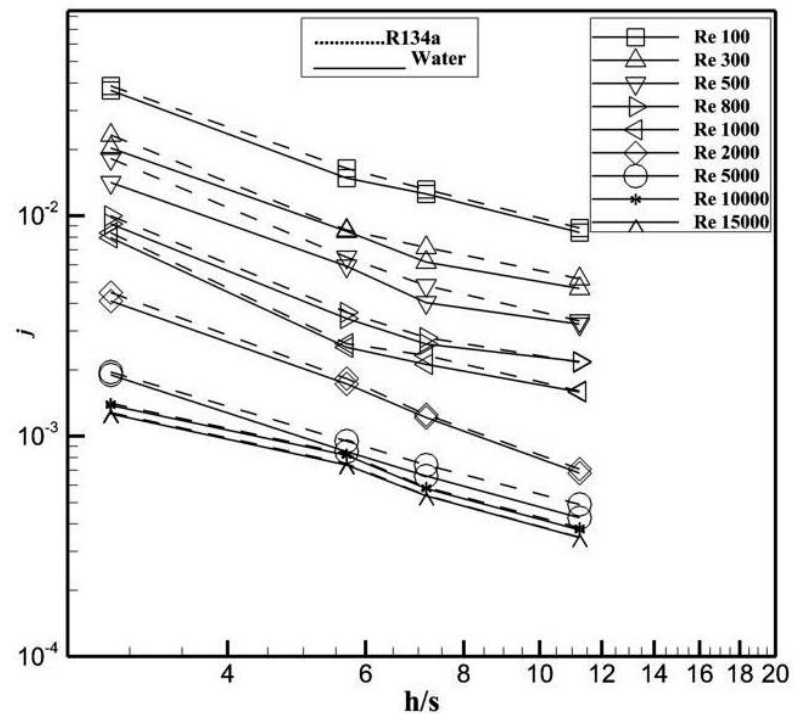

(a)

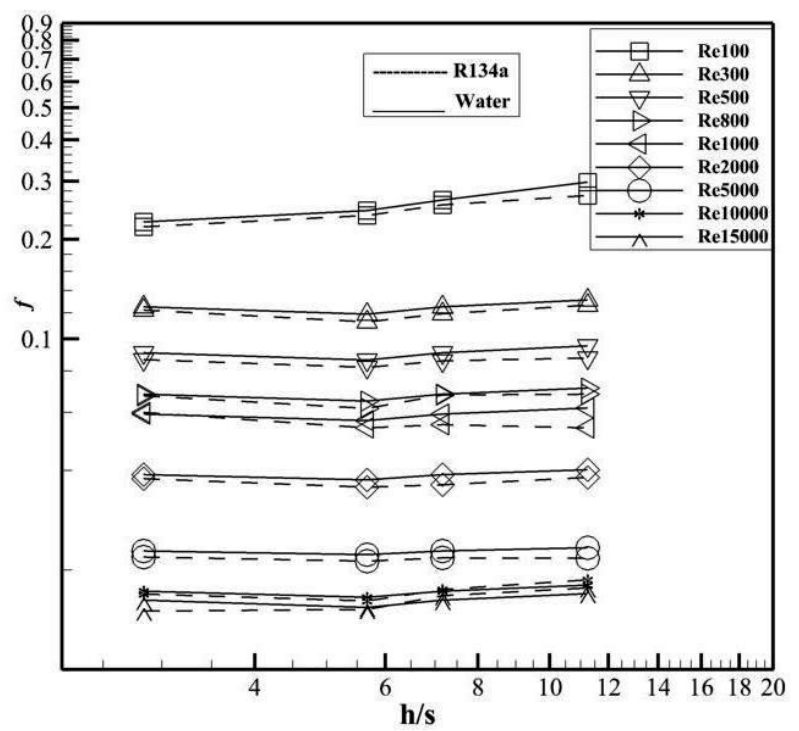

(b)

Fig. 6 (a) Effect of fin height/spacing on $\boldsymbol{j}$; (b) Effect of fin height/spacing on $f$.

with the increase of $\mathrm{h} / \mathrm{s}$ for constant Re. Colburn $j$ factor decreases with the increase of $\mathrm{h} / \mathrm{s}$. There is no significant variation of $f$ factor for both liquid water and liquid refrigerant R134a at constant Reynolds number. The difference is found less than $5 \%$ for both liquids. However the variation in $j$ factor is found for water and liquid refrigerant R134a.

\subsection{Effect of a/s Ratio on $j$ and $f$}

The friction factor $f$ and Colburn $j$ factors are plotted against the wave height (a)-to- fin spacing (s) ratio (a/s)

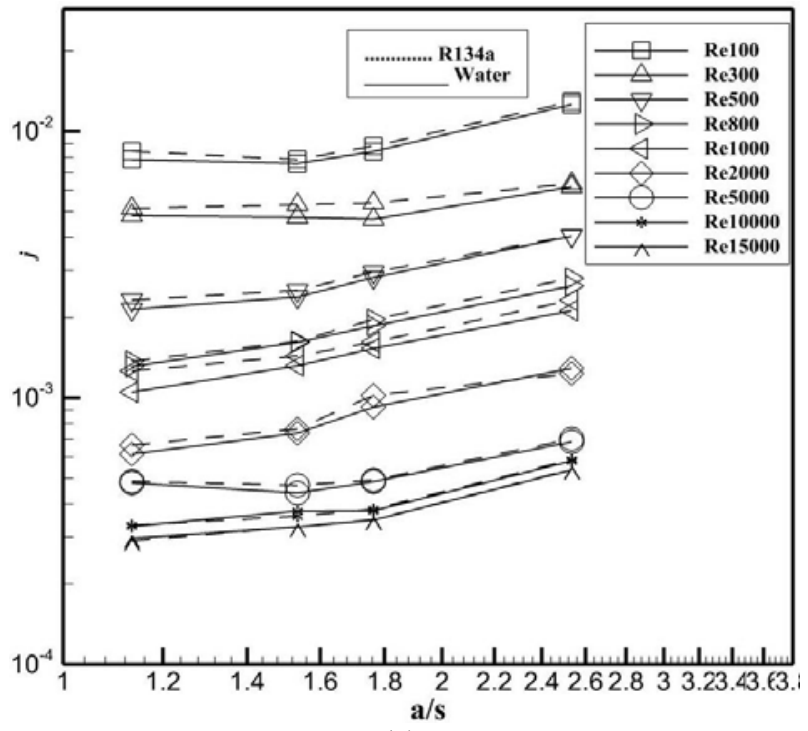

(a)

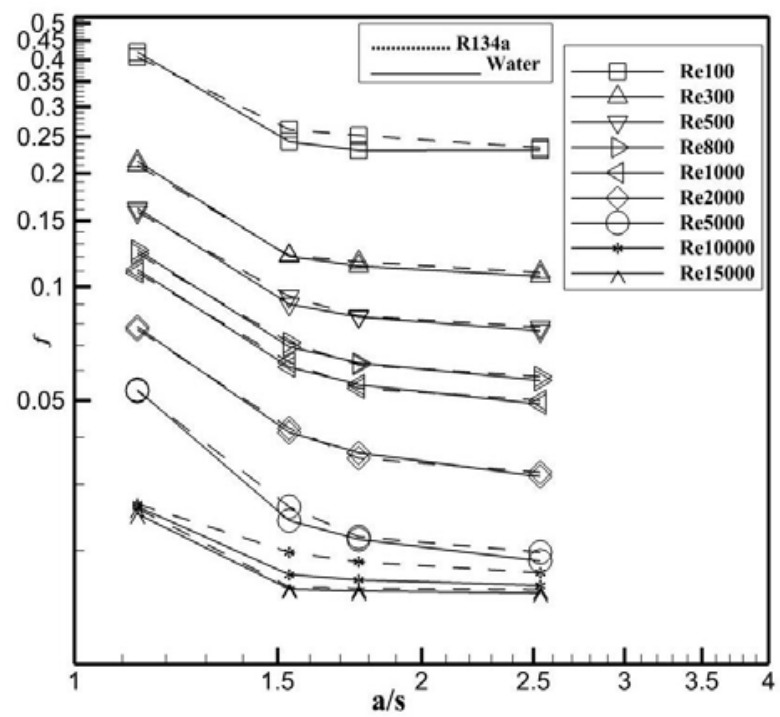

(b)

Fig. 7 (a) Effect of fin wave height/spacing on $j$; (b) Effect of fin wave height/spacing on $f$.

for varying Reynolds number in Fig. 7. Both $f$ and $j$ decrease with increase in $\mathrm{Re}$ as expected. Friction factor $f$ decrease with the increase of $\mathrm{a} / \mathrm{s}$ for constant $\mathrm{Re}$ Colburn $j$ factor increases with the increase of $\mathrm{a} / \mathrm{s}$. There is no significant variation of $f$ factor for both liquid water and liquid refrigerant R134a at particular Reynolds number. The difference is found less than $5 \%$ for both liquids. However the variation in $\mathrm{j}$ factor is found.

\subsection{Effect of $\lambda / a$ Ratio on $j$ and $f$}

The friction factor $f$ and Colburn $j$ factors are plotted 
against the wave length $(\lambda)$-to-wave height (a) ratio $(\lambda /$ a) for varying Reynolds number in Fig. 8. Both $f$ and $j$ decrease with increase in $\mathrm{Re}$ as expected. Friction factor $f$ decrease with the increase of $\lambda / \mathrm{a}$ for constant Re Colburn $j$ factor decreases with the increase of $\lambda / \mathrm{a}$ and even became constant high $\lambda /$ a value. There is no significant variation of $f$ factor for both liquid water and liquid refrigerant R134a at particular Reynolds number. The difference is found less than $5 \%$ for both liquids. However the variation in $j$ factor is found.

\subsection{Correlations for $j$ and $f$ Factors}

An extensive numerical study has been carried out on the heat transfer phenomena in plate fin surfaces with wavy fins. The $f$ vs. Re and $j$ vs. Re curves show significant non linearity as show in Figs. 6-8. The correlations have been expressed in terms of two separate equations over low and high Re regions along with dimensionless geometric parameters. The power law expressions have been used for determining the Colburn factor $j$ and friction factor $f$ as a function of the Reynolds number and dimensionless fin parameters. The use of these power law expressions is justified because variations in $f$ and $j$ with $\mathrm{Re}, \mathrm{h} / \mathrm{s}, \mathrm{a} / \mathrm{s}, \lambda / \mathrm{a}$ follow constant slope log-linear lines in both laminar and fully turbulent flow regions.

The Colburn factor $j$ and Fanning friction factor $f$ are functionally related to Re, h/s, a/s, $\lambda / \mathrm{a}$ and it can be represented in general form as:

$$
\text { or } j=C\left(R_{e}\right)^{a 0}(\alpha)^{a 1}(\beta)^{a 2}(\gamma)^{a 3}
$$

where $\mathrm{C}, \mathrm{a} 0, \mathrm{a} 1, \mathrm{a} 2$ and $\mathrm{a} 3$ are constants depends on the type of fin surface and geometry.

The $j$ vs. Re data for wavy fin surfaces show significant non linearity over the Reynolds number range $100 \leq \operatorname{Re} \leq 15,000$. There two separate equations have been proposed for the low and high Re regions as below. Also the variation in $j$ factor is found large for water and liquid refrigerant R134a. Hence, separate $j$ correlations have proposed for water and liquid R134a. This is due to significant effect of Prandtl number on heat transfer coefficient.

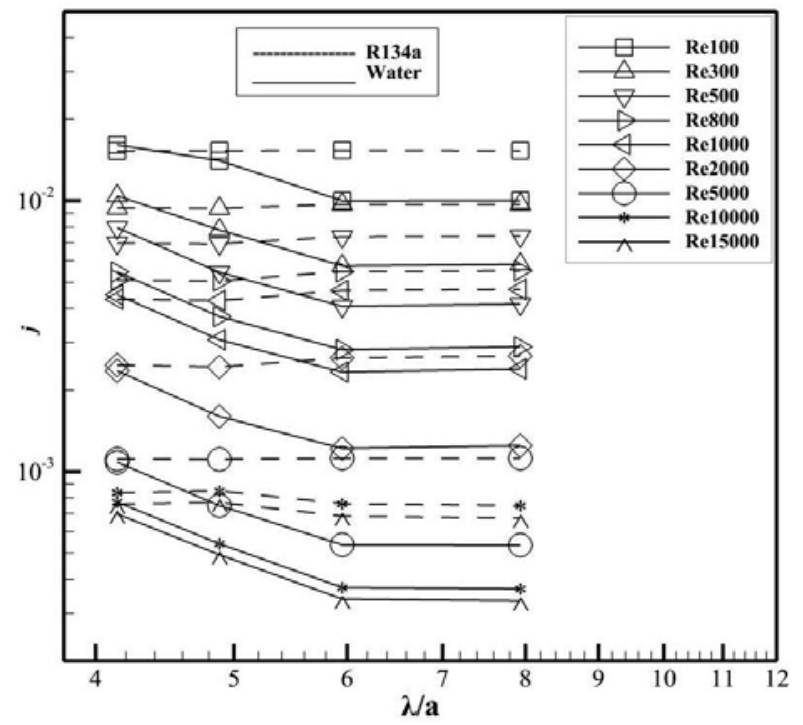

(a)

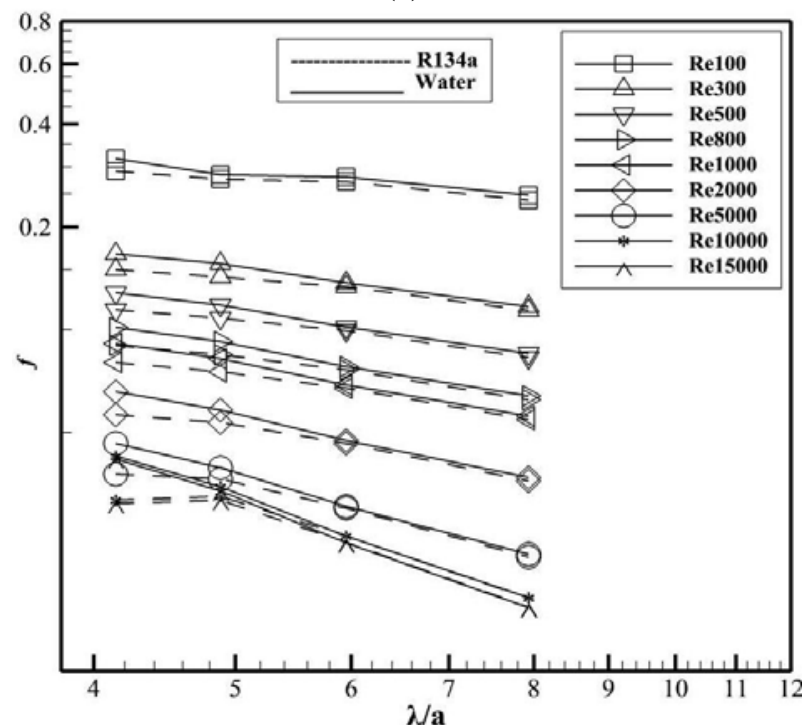

(b)

Fig. 8 (a) Effect of fin wave length/wave height on $j$; (b) Effect of fin wave length/wave height on $f$.

$$
\begin{gathered}
j=2.989 R e^{-0.54241} \alpha^{-0.72276} \beta^{-0.83914} \gamma^{-0.7588} \\
\text { for } 100 \leq \operatorname{Re} \leq 1000 \\
j=3.245 R e^{-0.66388} \alpha^{-0.53614} \beta^{-0.80626} \gamma^{-0.6346} \\
\text { for } 1000 \leq \operatorname{Re} \leq 15000 \\
\quad \text { for } 100 \leq \operatorname{Re} \leq 1,000 \\
\text { for } 1,000 \leq \operatorname{Re} \leq 15,000
\end{gathered}
$$

Eqs. 13 and 14 predict the $j$ factor for R134a and Eqs. 15 and 16 predict for the water. The above correlations predict the $99 \%$ of $j$ data for laminar regions and $96 \%$ 
of the $j$ data for the turbulent regions.

Similarly the $f$ vs. Re data for wavy fin surfaces show significant non linearity over the Reynolds number range $100 \leq \mathrm{Re} \leq 15,000$. Those two separate equations have been proposed for the low and high Re regions as below. The deviation between $f$ data of water and liquid R134a is less than 5\%. Hence, single correlations have proposed for both fluids. Prandtl number was found to have little effect on the Fanning friction factor:

$$
\begin{gathered}
f=18.607 R e^{-0.59381} \alpha^{-0.088954} \beta^{-0.46976} \gamma^{-0.92621} \\
\text { for } 100 \leq \operatorname{Re} \leq 1,000 \\
f=24.413 R e^{-0.46532} \alpha^{-0.226497} \beta^{-0.94256} \gamma^{-1.70937} \\
\text { for } 1000 \leq \operatorname{Re} \leq 15,000
\end{gathered}
$$

The above correlations predict the $99 \%$ of $f$ data for laminar regions and $96 \%$ of the $f$ data for the turbulent regions.

\section{Conclusions}

This paper presents the heat transfer and pressure drop correlations for wavy fins with water and R134 a as working medium. This type fins widely used in aerospace and automobile industries. The expressions provided for the heat transfer coefficient in terms Colburn $j$ factor and friction factor $f$ allow the computations for all values of Reynolds number, including the laminar and turbulent regions. The generalized correlations are developed for smooth wavy fins taking all geometrical parameters into account for entire range of operations in industry. In addition the effects of various geometrical parameters are presented as parametric studies. These expressions are well formed in the laminar and fully turbulent regions, since they can be considered as the standard expressions modified by correction factors. The proposed correlations can be used by heat exchanger designers and can reduce the number of tests and modifications of the prototype to a minimum for similar applications. Single $f$ correlations and separate $j$ correlations have proposed for both water and liquid refrigerant $\mathrm{R} 134 \mathrm{a}$, since the variation of $f$ varies less than $5 \%$ and where as $j$ varies up to $15 \%$. The $j$ vs. Re $\& f$ vs. Re data for wavy fin surfaces show significant non linearity over the Reynolds number range $100 \leq \mathrm{Re}$ $\leq 15,000$. Two separate equations have been proposed for the low and high Re regions i.e. Between $\mathrm{Re}$ of 100-1,000 and Re of 1,000-15,000. Colburn $j$ factor and Fanning friction factor $f$ Correlations have proposed in terms Re and geometry parameters $(\mathrm{h} / \mathrm{s}, \mathrm{a} / \mathrm{s}$, $\lambda / a)$ for water and liquid refrigerant R134a in the present study.

\section{Acknowledgements}

The authors acknowledge Aeronautical Development Agency, Bangalore and National Institute of technology, Surathkal, Mangalore for allowing to publish this paper.

\section{References}

[1] Shah, R. K. and London, A. L. 1968. "Offset Rectangular Plate-Fin Surfaces-Heat Transfer and Flow Friction Characteristics." ASME J. Eng Power 90: 218-28.

[2] Kays, W. H. and London, A. L. 1984."Compact Heat Exchangers." $3^{\text {rd }}$ edition, McGraw Hill, New York.

[3] Joshi, H. M. and Webb, R. L. 1987. "Heat Transfer and Friction in the Offset Strip Fin Heat Exchanger." Int. J. Heat Mass Transfer 30: 69-84.

[4] Wieting, A. R. 1975. "Empirical Correlation for Heat Transfer and Fow Friction Characteristics of Rectangular Offset-Fin Plate Fin Heat Exchangers." ASME J. Heat Transfer 97: 488-90.

[5] Manglik, R. M. and Bergles, A. E. 1995. "Heat Transfer and Pressure Drop Correlations for the Rectangular off Strip Fin Compact Heat Exchanger." Experimental Thermal and Fluid Science 10: 171-80.

[6] Ranganayakulu, C., and Pallavi, P. 2011 "Development of Heat Transfer Coefficient and Friction Factor Correlations for Offset Fins Using CFD." International Journal of Numerical Methods for Heat \& Fluid Flow 21 (8): 935-51.

[7] Zhang,J., Jaydeep, K. and Manglik, R. M. 2004. "Effect of Fin Waviness and Spacing on the Lateral Vortex Structure and Laminar Heat Tansfer in Wavy-Plate-Fin Cores." International Journal of Heat and Mass Transfer 47: 1719-30.

[8] Awad, M. M, and Muzychka, K. S. 2011. "Models for Pressure Drop and Heat Transfer in Air Cooled Compact Wavy Fin Heat Exchangers." Journal of Enhanced Heat Transfer 18 (3): 191-207. 
[9] Hu, S. and Herold, K. E. 1995. "Prandtl Number Effect on Offset Fin Heat Exchanger Performance: Predictive Model for Heat Transfer and Pressure Drop." Int. J. Heat Mass Transfer 38 (6): 1043-51.

[10] Hu, S. and Herold, K. E. 1995. "Prandtl Number Effect on Offset Fin Heat Exchanger Performance: Experimental Results." Int J Heat Mass Transfer 38: 1053-61.

[11] Ranganayakulu, C., Mersmann, I. and Kabelac, S. 2013. "Boiling of R134a in a Plate-Fin Heat Exchanger Having Offset Fins." In Proceedings of the $22^{\text {th }}$ National and $11^{\text {th }}$ International ISHMT-ASME Heat and Mass Transfer Conference, 28-31.

[12] Kim, B. and Sohn, B. 2006. "An Experimental Study of Flow Boiling in a Rectangular Channel with Offset Strip Fins." International Journal of Heat and Fluid Flow 27: 514-21.

[13] Maiti, D. K. 2002. "Heat Transfer and Flow Friction Characteristics of Plate-Fin Heat Exchanger Surfaces-a Numerical Study." Ph.D. thesis, IIT Kharagpur.

[14] Ranganayakulu, C., Ismail, L. S., Rao, V. V. and Rajeshwar, S. 2008. "Optimization of Wavy Fin Parameters in Compact Heat Exchangers." In Proceedings of XIX National Heat and Mass Transfer \& VIII ISHMT-ASME Heat and Mass Transfer Conference, January.
[15] Chen, J. C. 1966. "Correlation for Boiling Heat Transfer to Saturated Fluids in Convective Flow." I \& EC Process Design and Development 5 (3): 322-39.

[16] Versteeg, H. K. and Malalasekera, W. 1995. "An Introduction to Computational Fluid Dynamics." The Finite Volume Method Prentice Hall, New York, 62-146.

[17] Anderson, J. D. $1995 . \quad$ "Computational Fluid Dynamics-The Basic with Applications." McGraw-Hill Companies, Inc, New York.

[18] Patankar, S. V. 1980. "Numerical Heat Transfer and Fluid Flow, Hemisphere Series on Computational Methods in Mechanics and Thermal Science." Taylor and Francis, 79-86.

[19] Patankar, S. V. Liu, C. H. and Sparrow, E. 1977. "Fully Developed Flow and Heat Transfer in Ducts Having Stream Wise-Periodic Variations of Cross Sectional Area." Journal of Heat transfer 99: 180-6.

[20] Welmer, R. F. and Hartzog, D. G. 1977. "Effect of Maldistribution on the Performance of Multistream Multipassage Heat Exchangers." Adv Cryo Engg 18: 52-64.

[21] Haseler, L. E. 1993. "Performance Calculation Methods for Multi Stream Plate Fin Heat Exchangers Heat Exchangers-Theory and Practice." McGraw Hill, New York, 405-506. 